\title{
Chemical Syntheses under Pressure
}

$\mathrm{B}^{\mathrm{Y}}$ $Y$ invitation of the president, Prof. G. T. Morgan, an ordinary scientific meeting of the Chemical Society was held at the Chemical Research Laboratory on Thursday, June 7, when three papers were contributed illustrating recent researches in the Laboratory on syntheses of organic substances under pressure.

Mr. R. Taylor described the circulatory plant (afterwards seen in operation) employed in studying condensations between carbon monoxide and hydrogen at $400^{\circ}$ and under 250 atmospheres in the presence of various catalysts, with a rate of circulation of about 80 litres of compressed gas per hour. In these researches, attention has been directed specially to the production of alcohols other than methyl alcohol (methanol). With a catalyst consisting of cobalt sulphide mixed with oxides of copper and manganese, an optimum yield of ethyl alcohol was obtained, although this synthesis was always accompanied by large amounts of methane. Systematic fractionation showed that more than ninety per cent of a product (b.p. $783^{\circ}$ ) consisted of alcohols with unbranched chains, among which $n$-hexyl and $n$-heptyl alcohols were identified. Branched chain products were represented by isobutyl alcohol, 2 -methylbutanol and 2-methylpentanol. A manganese-chromium catalyst strongly alkalised by rubidia gave a product in which all alcohols identified above the $\mathrm{C}_{3}$ compound had branched chains. Another catalyst containing cobalt and strong alkali gave a mixture of branched and straight chain alcohols.

The chemical reactions involved in these syntheses were discussed, and successive aldolisation and hydrogenation were suggested as the directive mechanism. For straight chain alcohols, acetaldehyde (a product actually identified in these condensations) must be present to provide the active hydrogen for aldolisation, and its condensation with propaldehyde would eventually lead to $n$-amyl alcohol. When propaldehyde furnishes the active hydrogen, the final product is 2 -methylpentanol. It may become possible to build up alcohols with a predetermined number of carbon atoms.

Dr. D. V. N. Hardy indicated an alternative mechanism by which it is supposed that carbon monoxide is added directly to alcohols with production of acids, which may then be reduced to aldehydes and alcohols. The condensations between methyl alcohol and carbon monoxide have been studied at $320^{\circ}-340^{\circ}$ under a pressure of 150 atmospheres in the presence of phosphoric acid. The gas was circulated at a rate of 2 cubic metres per hour, and $120 \mathrm{gm}$. of methyl alcohol vapour was introduced into the system during the same period. Acetic acid and methyl acetate were obtained, together with an oily layer containing high boiling hydrocarbons from which hexamethylbenzene was isolated. When 2 per cent by weight of copper phosphate was added to the phosphoric acid, this oily layer was no longer formed and larger proportions of acetic acid and its methyl ester were obtained. Some dimethyl ether was identified, but as it did not accumulate in the system it may be regarded as a reagent. These experiments favour the view that a methylene radical is produced which unites with carbon monoxide to form ketene, this reactive compound being then hydrated and methyl. ated to acetic acid and methyl acetate respectively.

Dr. D. D. Pratt discussed the use made of autoclaves in practically all research sections of the Laboratory. These autoclaves, which have been constructed in the laboratory workshop, have capacities ranging from 50 c.c. to 10 litres; they are capable of withstanding pressures of 200 atmospheres at temperatures up to $450^{\circ}$.

Phenols, aromatic hydrocarbons and bases undergo carboxylation when condensed with carbon dioxide under pressure in presence of catalysts such as aluminium and zinc chlorides.

High pressure aminations of alcohols and phenols have led to significant results. Resorcinol and orcinol heated to $200^{\circ}$ with aqueous ammonia give rise to $m$-aminophenol and 5-amino- $m$-cresol respectively. In similar circumstances resorcinol and ethylamine furnish $m$-ethylaminophenol, an important colour intermediate.

Autoclave experiments have been made on the reactions between hydroxylic compounds and ammonium chloride. At $300^{\circ}$, ethyl alcohol gives a mixture of mono-, di-, and tri-ethylamines, separated by fractionation through a Dufton column. At $320^{\circ}$ $350^{\circ}$, phenols are converted into a mixture of primary and secondary amines. This amination, which is particularly successful with $m$-cresol and symmetrical xylenol, is of technical importance, since the resulting $m$-toluidine and $1: 3: 5$-xylidine are not readily obtained by successive nitration and reduction from toluene and $m$-xylene. In these aminations of phenolic homologues, the effect of orientation in the aromatic nucleus is plainly discernible. In the diphenyl series the ammonium chloride reaction on 2 -hydroxydiphenyl and $2: 2^{\prime}$-dihydroxydiphenyl leads respectively to $o$-xenylamine and carbazole, another important colour intermediate.

At the conclusion of the meeting, the visitors were conducted through the laboratories, in which exhibits and demonstrations had been arranged illustrating the following researches:-

Synthetic production of methyl and other alcohols from carbon monoxide and hydrogen, and the synthesis of acetic acid from methyl alcohol and carbon monoxide. For the characterisation of higher aldehydes and alcohols, it is necessary to have authentic specimens of well-crystallised derivatives, and a collection of these products was on view.

In the tar section there were demonstrations of shirlacrol, a new wetting agent used in mercerising cotton, and of the extraction of catechol and resorcinol from industrial liquors. The identification of tar constituents involves the synthesis of higher phenols and complex aromatic hydrocarbons. Dis tinctive specimens of such products were exhibited together with fuel oils derived from the hydrogena. tion of tars. In the road tar section a large-scale plant for the separation of the crystalloid and resinoid constituents of tars was in actual operation.

The allied sections of chemotherapy and synthetic resins were illustrated by comprehensive collections.

Inorganic chemistry was represented by researches on the aerial and immersed corrosion of metals, the production of base exchange materials from English clays and the isolation of rarer metals, such as germanium and rhenium, from British minerals. The exhibits arranged by the microbiological and dental investigation sections were also greatly appreciated by the visitors, who numbered about 260 . 